Nonlinear Dispersive Instabilities in Kelvin-Helmholtz Magnetohydrodynamic Flows

This content has been downloaded from IOPscience. Please scroll down to see the full text. 2003 Phys. Scr. 67340

(http://iopscience.iop.org/1402-4896/67/4/012)

View the table of contents for this issue, or go to the journal homepage for more

Download details:

IP Address: 146.175.11.111

This content was downloaded on 09/12/2013 at 14:58

Please note that terms and conditions apply. 


\title{
Nonlinear Dispersive Instabilities in Kelvin-Helmholtz Magnetohydrodynamic Flows
}

\author{
A. H. Khater ${ }^{1,2, *}$, D. K. Callebaut ${ }^{2}$ and A. R. Seadawy ${ }^{1}$ \\ ${ }^{1}$ Mathematics Department, Faculty of Science, Cairo University, Beni-Suef, Egypt \\ ${ }^{2}$ Department Natuurkunde, UIA, Universiteit Antwerpen, B-2610 Antwerpen, Belgium \\ Received July 11, 2002; revised version November 15, 2002; accepted November 21, 2002

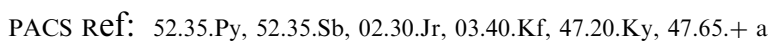

\begin{abstract}
In this paper a weakly nonlinear theory of wave propagation in superposed fluids in the presence of magnetic fields is presented. The equations governing the evolution of the amplitude of the progressive waves are reported. The nonlinear evolution of Kelvin-Helmholtz instability (KHI) is examined in $2+1$ dimensions in the context of magnetohydrodynamics (MHD). We study the envelope properties of the $2+1$ dimensional wave packet. We converted the resulting nonlinear equation for the evolution of the wave packets in a $2+1$ dimensional nonlinear Schrödinger (NLS) equation by using the function transformation method into a sine-Gordon equation, which depends only on one function, $\zeta$. We obtained rather general classes of solutions of the equation in $\zeta$ which leads to rather general soliton solutions of the $2+1$ dimensional NLS equation. This result contains interesting specific solutions such as $N$ multiple solitons, propagational breathers and quadratic solitons.
\end{abstract}

\section{Introduction}

Under certain conditions the interface of two fluids in motion develops an instability known as the KelvinHelmholtz instability (KHI). This instability has many applications in astrophysics, space physics, plasma dynamics, gas and fluid flows. The development of this instability determines the dynamic processes in the hoses of spiral galaxies and in the jet bands in radiogalaxies [1], as well as the processes in the magnetic break zone [2] and at the layer boundaries of the stratified atmosphere of planets and commetary tails [3], sheared plasma flow such as the stability of the interface between the solar wind and the magnetosphere [4-6], interaction between the adjacent streams of different velocities in the solar wind [7] and laboratory experiments aiming to study the nonlinear saturated stage of the cross-field electrostatic KHI in a strongly magnetized plasma [8-13]. In application to plasma problems, the inhomogeneous plasma flux instability in crossed electric and magnetic fields [2,14-16] is an important particular case. In the geospace-magnetosphere, observations demonstrate that there are various nonlinear waves in the upper ionosphere [17] and auroral region [18]. Wu et al. [19-20] studied solitary waves and discussed the possibility of applying their results to nonlinear waves observed by the scientific satellite Freja in the upper ionosphere [21].

Michael [22] considered the stability of two inviscid, incompressible and perfectly conducting fluids, moving with uniform velocities parallel to their interface and in which parallel magnetic fields along the direction of streaming were present. He showed that, within the

\footnotetext{
*Corresponding author. e-mail: khater_ah@hotmail.com
}

framework of the linear theory, the magnetic fields have a stabilizing effect on the configuration. The linear theory governing the KHI in MHD when two superposed fluids move with uniform speeds was developed by Chandrashekhar [23]. Drazin [24], Nayfeh and Saric [25] and Weissman [26] investigated the nonlinear aspects of such instability in ideal fluids. In MHD, Kant and Malik [27] and Malik and Singh [28] studied the nonlinear evolution of wave packets for such a type of stability. They reported that for subcritical MHD flows where the velocity difference $U$ is smaller than the critical velocity $U_{c}$, the dynamical equation yielding the amplitude is a nonlinear Schrödinger equation (NLS). Kant and Malik [27] also showed that a wave train of constant amplitude is unstable against modulations in the presence of a magnetic field. Using inverse-scattering transforms (IST), Zakharov and Shabat [29] obtained the solution of a NLS equation in $1+1$ dimensions. Their analysis reveals that smooth initial data lead to localized soliton solutions, and is related to the terminal state of the modulational instability. Moreover Khater et al. [30] used the Bäcklund transformations to obtain classes of exact solutions.

For postcritical values of $U$, the amplitude evolution for supercritical MHD flows was described by a nonlinear Klein-Gordon equation. From this equation, Malik and Singh [28] derived kink, antikink and solitary-wave solutions. If one neglects the spatial variation in amplitude then the Klein-Gordon equation gives rise to exponential decay, subcritical, supercritical and explosive instabilities. However, considerable effort has been devoted to the phenomenon of self-focusing and collapse; in nonlinear optics by Zakharov and Synakh [31], in plasma physics by Zakharov [32] and in two-layer Kelvin-Helmholtz flows by Gibbon and McGuinness [33]. Singh, Khosla and Malik [34] derived the dynamical equations governing the amplitudes of the fundamental mode and the secondharmonic mode, and they integrated these equations numerically by employing the fourth-order Runge-Kutta method. In conservative systems, solutions are found by direct integration, suitable transformation or substitution, or other ad hoc techniques. The original PDE could be solved also with more sophisticated methods such as the Hirota bilinear technique [35], truncated Painlevé expansion [36], direct algebra methods [37-38] and the like. Other PDEs are no longer that easy to solve. For instance, ingenious transformations are needed to obtain closed form solutions to the $\mathrm{KdV}$-Burgers equation [39-40], despite the simplicity of this equation. 
In this paper, a function transformation is introduced and applied to obtain plane soliton solutions, $N$ multiple soliton solutions, the propagational breathers and the quadratic solitons, which contain spherical, ellipsoidal and hyperboloidal solitons.

The paper is organized as follows: In Section 2, the basic equations and the boundary conditions governing the problem together with the multiple scale method are sketched. In Section 3, nonlinear focusing, self-focusing and the standard NLS equation in $2+1$ dimensions in elliptic and hyperbolic cases are briefly reported. In Section 4 , we find general soliton solutions to the standard NLS equation in $2+1$ dimensions in elliptic and hyperbolic cases. We applied the function transformation method, then the resulting nonlinear equation for the evolution of the wave packets in $2+1$ dimensions, i.e. the NLS equation, is transformed into a sine-Gordon equation, which depends only on one function, $\zeta$. We obtained a general solution of the equation in $\zeta$ which leads to a general soliton solution of the $2+1$ dimensions NLS equation. The latter contains some interesting specific solutions such as $N$ multiple solitons, propagational breathers and quadratic solitons. In Section 5, we discuss the stability for the soliton solutions. Section 6 yields conclusions.

\section{Problem formulation}

Consider two superposed fluids separated by the interface $z=0$. The fluids are semi-infinite, inviscid, incompressible and perfectly conducting with surface tension present at the boundary. A fluid with density $\rho_{1}$ occupies the half-space $z<0$ whereas $z>0$ ( $z$ is the vertical axis) is occupied by a fluid with density $\rho_{2}$. The upper and lower fluids are moving with uniform velocities $U^{(1)}$ and $U^{(2)}$ along the $x$ axis. The system is subjected to a uniform tangential magnetic field $\boldsymbol{H}(H, 0,0)$ permeating both fluids in the presence of a gravitational acceleration $g(0,0,-g)$. We examine the propagation of weakly nonlinear wave packets of small but finite amplitude, with principal direction along the $x$-axis, although modulation in both $x$ and $y$ directions is allowed. The basic equations describing this MHD flow are

$$
\begin{aligned}
& \frac{\partial \boldsymbol{u}^{(i)}}{\partial t}+\left(\boldsymbol{u}^{(i)} \cdot \nabla\right) \boldsymbol{u}^{(i)}=-\nabla \Pi^{(i)}+\frac{1}{\rho^{(i)}}\left(\boldsymbol{h}^{(i)} \cdot \nabla\right) \boldsymbol{h}^{(i)}+\boldsymbol{g}, \\
& \frac{\partial \boldsymbol{h}^{(i)}}{\partial t}=\left(\boldsymbol{h}^{(i)} \cdot \nabla\right) \boldsymbol{u}^{(i)}-\left(\boldsymbol{u}^{(i)} \cdot \nabla\right) \boldsymbol{h}^{(i)}, \\
& \nabla \cdot \boldsymbol{u}^{(i)}=0, \quad \nabla \cdot \boldsymbol{h}^{(i)}=0 .
\end{aligned}
$$

Here $i=1$ and 2 correspond to the lower and upper regions respectively. The fluid velocity is $\boldsymbol{u}^{(i)}=\left(u^{(i)}, v^{(i)}, w^{(i)}\right)$, the magnetic field is $\boldsymbol{h}^{(i)}$ (including $\sqrt{\mu}$ with $\mu$, the magnetic permeability, taken as constant) and the total pressure in region $(i)$ is $\rho^{(i)} \Pi^{(i)}=P^{(i)}+\frac{1}{2}\left|\boldsymbol{h}^{(i)}\right|^{2}$, where $\frac{1}{2}\left|\boldsymbol{h}^{(i)}\right|^{2}, P^{(i)}$ and $t$ stand as usual for the magnetic pressure, gas pressure and time respectively. In some papers [34] one has used for the magnetic field $\left(\rho^{(i)}\right)^{1 / 2} \boldsymbol{h}^{(i)}$ instead. This avoids the factor $1 / \rho^{(i)}$ in Eq. (1), but this factor has then to appear in the boundary condition related to the conservation of flux in Eq. (3). We have adapted for this aspect and hence our expressions for the constants (see Eq. (11)) differ somewhat from theirs.

The boundary conditions at the free interface are:

(a) The kinematic boundary condition

$$
\frac{\partial \eta}{\partial t}+\left(\boldsymbol{u}^{(i)} \cdot \nabla\right) \eta=w^{(i)} \quad \text { at } \quad z=\eta, \quad(i=1,2) .
$$

(b) The continuity of the normal component of the magnetic field

$$
\boldsymbol{n} \cdot \boldsymbol{h}^{(1)}=\boldsymbol{n} \cdot \boldsymbol{h}^{(2)}
$$

where $\boldsymbol{n}$ is a normal to the boundary and $\eta$ denotes the elevation of the free surface above the unperturbed level $z=\eta(x, y, t)$; thus

$$
h_{z}^{(1)}-h_{x}^{(1)} \frac{\partial \eta}{\partial x}-h_{y}^{(1)} \frac{\partial \eta}{\partial y}=h_{z}^{(2)}-h_{x}^{(2)} \frac{\partial \eta}{\partial x}-h_{y}^{(2)} \frac{\partial \eta}{\partial y} .
$$

(c) The continuity of the normal stress at the perturbed surface $z=\eta(x, y, t)$ is

$$
\begin{aligned}
\Pi^{(1)}- & \rho \Pi^{(2)}-(1-\rho) g \eta \\
= & \frac{T}{\rho_{1}}\left[\nabla^{2} \eta\left(1+(\nabla \eta)^{2}\right)-\frac{1}{2} \nabla \eta \cdot \nabla(\nabla \eta)^{2}\right] \\
& \times\left(1+(\nabla \eta)^{2}\right)^{-3 / 2}
\end{aligned}
$$

where $T$ is the coefficient of surface tension and $\rho=\rho_{2} / \rho_{1}$. This equation may be rewritten as

$$
\begin{aligned}
\Pi^{(1)}- & \rho \Pi^{(2)}-(1-\rho) g \eta \\
= & \frac{T}{\rho_{1}}\left[\frac{\partial^{2} \eta}{\partial x^{2}}+\frac{\partial^{2} \eta}{\partial y^{2}}+\left(\frac{\partial \eta}{\partial y}\right)^{2} \frac{\partial^{2} \eta}{\partial x^{2}}+\left(\frac{\partial \eta}{\partial x}\right)^{2} \frac{\partial^{2} \eta}{\partial y^{2}}\right. \\
& \left.-2 \frac{\partial^{2} \eta}{\partial x \partial y} \frac{\partial \eta}{\partial x} \frac{\partial \eta}{\partial y}\right]\left[1+\left(\frac{\partial \eta}{\partial x}\right)^{2}+\left(\frac{\partial \eta}{\partial y}\right)^{2}\right]^{-3 / 2} .
\end{aligned}
$$

We wish to examine the nonlinear stability problem posed by Eqs. (1) to (6). In order to describe nonlinear interactions of small but finite-amplitude waves, we employ the multiple scales method [41]. To do this, we introduce the variables

$x_{n}=\varepsilon^{n} x, \quad y_{n}=\varepsilon^{n} y, \quad t_{n}=\varepsilon^{n} t(n=0,1,2, \ldots, N)$,

and expand the various perturbed quantities in the following series:

$$
\begin{aligned}
\eta(x, y, z, t)= & \sum_{n=0}^{N} \varepsilon^{n} \eta_{n}\left(x_{0}, x_{1}, \ldots, x_{n} ; y_{0}, y_{1}, \ldots, y_{n}\right. \\
& \left.\times z ; t_{0}, t_{1}, \ldots, t_{n}\right)+O\left(\varepsilon^{N+1}\right),
\end{aligned}
$$

and similarly for $\boldsymbol{u}(x, y, z, t)$ and $\boldsymbol{h}(x, y, z, t)$. Here $\varepsilon$ represents a small parameter characterizing the steepness ratio of the wave. 
For the problem under investigation, it is sufficient to take $N=3$ as far as the lowest significant order is concerned. Equations (1) to (6) require a priori information of the displacement $\eta(x, y, t)$ at the perturbed surface. To circumvent this problem, we use Taylor series expansions of various quantities about $z=0$. On substituting expressions (8) and the corresponding derivatives into (1)-(3) and the transformed boundary conditions (4)-(6), we obtain on equating the coefficients of terms of equal powers in $\varepsilon$, a linear and successive nonlinear partial differential equations (PDEs) of various orders. The problem may then be solved for any order with the knowledge of the solutions of all the previous orders.

\section{Nonlinear focusing}

We shall now investigate the most dramatic consequence for the wave packets: nonlinear focusing. Self-focusing provides a mechanism by which a field of relatively smallamplitude waves produces a local region in which the amplitudes become unbounded in a finite time. The point where the field becomes singular is called the 'focus'. Focusing is a potentially important mechanism in the redistribution of energy within the spectrum.

Consider the propagation of nonlinear wave packets of small but finite amplitudes propagating in the principal direction along the $x$-axis with modulation allowed in both the $x$ and $y$ directions.

Following Kant and Malik [27]; Malik and Singh [28]; Pusri and Malik [42]; Singh, Khosla and Malik [34]; and using the multiple scales method, (1)-(6) yield the linear dispersion relation

$$
D(\omega, K)=-\left(\alpha_{1} \sigma_{1}^{2}+\alpha_{2} \sigma_{2}^{2}\right)+l^{2} V_{A}^{2}+K^{3}+K=0
$$

where

$$
\begin{aligned}
& \alpha_{1}=\frac{\rho_{1}}{\rho_{1}+\rho_{2}}, \quad \alpha_{2}=\frac{\rho_{2}}{\rho_{1}+\rho_{2}}, \quad V_{A}^{2}=\frac{2 \mu H^{2}}{\rho_{1}+\rho_{2}}, \\
& \sigma_{i}=l U^{(i)}-\omega, \quad K^{2}=l^{2}+m^{2}, \quad i=1,2 .
\end{aligned}
$$

All the perturbed quantities are normalized with respect to the characteristic length $\left[T /\left(\rho_{1}-\rho_{2}\right) g\right]^{1 / 2}$ and the characteristic speed $\left[\left(\rho_{1}-\rho_{2}\right) g T /\left(\rho_{1}+\rho_{2}\right)^{2}\right]^{1 / 4}$. The quantities $l$ and $m$ correspond to the components of the wave vector along the $x$ and $y$ directions respectively and $V_{A}$ is the Alfvén wave speed corresponding to the averaged density. Equation (9) furnishes the critical velocity difference $U_{c}$ at $K=K_{c}=1$, as was developed by Chandrashekhar [23]:

$U_{c}=\left(\frac{2+V_{A}^{2}}{\alpha_{1} \alpha_{2}}\right)^{1 / 2}$.

In the dispersion relation (9), we observe the magnetic field having a stabilizing influence on the wave motion, like the surface tension. Since we are interested primarily in studying the amplitude modulation of the progressive wave, we assume that $U^{(1)}-U^{(2)}<U_{c}$ and proceed to obtain the second-order solution. This second-order solu- tion is uniformly valid subject to the solvability condition and the non-secularity condition. For the second-order problem it reads:

$-D_{\omega} \frac{\partial A}{\partial t_{1}}+D_{l} \frac{\partial A}{\partial x_{1}}+D_{m} \frac{\partial A}{\partial y_{1}}=0$,

where $D_{\omega}, D_{l}$ and $D_{m}$ are the partial derivatives of the dispersion relation (9) with respect to frequency $\omega$ and wave numbers $l$ and $m$, respectively. The amplitude $A$ of the progressive wave is a function of the faster scales $\left(x_{1}\right.$, $\left.x_{2} ; y_{1}, y_{2} ; t_{1}, t_{2}\right)$. Introducing

$V_{l}=-\frac{D_{l}}{D_{\omega}}, \quad V_{m}=-\frac{D_{m}}{D_{\omega}}$

and substituting into (12), we get

$\frac{\partial A}{\partial t_{1}}+V_{l} \frac{\partial A}{\partial x_{1}}+V_{m} \frac{\partial A}{\partial y_{1}}=0$.

The quantities $x_{1}$ and $y_{1}$ are the fast space scales, while $V_{l}$ and $V_{m}$ represent a kind of group velocities along the $x$ and $y$ directions respectively, given by

$D_{\omega}=2\left(\alpha_{1} \sigma_{1}+\alpha_{2} \sigma_{2}\right), \quad D_{m}=\frac{m}{K}\left(3 K^{2}+1\right)$,

$D_{l}=\frac{-2}{K}\left(\alpha_{1} U^{(1)} \sigma_{1}+\alpha_{2} U^{(2)} \sigma_{2}\right)+\frac{l}{K}\left(3 K^{2}+1\right)+\frac{2 l}{K} V_{A}^{2}$,

then we have

$$
\begin{aligned}
& V_{l}=\frac{2\left(\alpha_{1} U^{(1)} \sigma_{1}+\alpha_{2} U^{(2)} \sigma_{2}\right)-\frac{l}{K}\left(3 K^{2}+1\right)-2 l V_{A}^{2}}{2\left(\alpha_{1} \sigma_{1}+\alpha_{2} \sigma_{2}\right)}, \\
& V_{m}=\frac{-m\left(3 K^{2}+1\right)}{2 K\left(\alpha_{1} \sigma_{1}+\alpha_{2} \sigma_{2}\right)} .
\end{aligned}
$$

It is interesting to remark here that, unlike ordinary gravity waves, for Kelvin-Helmholtz flows the group velocities along the $x$ and $y$ directions are not parallel to the wave velocities. Furthermore, the asymptotic expansions break down for $K^{2}=\frac{1}{2}$ (the breakdown at $K^{2}=\frac{1}{2}$ in Eq. (17) is due to $Q_{1}$ ), which corresponds to a second-harmonic resonance. At resonance, the fundamental and secondharmonic modes propagate with the same phase speed, resulting in energy transfer from one mode to another. It was observed that e.g. sea waves occurred at wind velocities far below $U_{c}(=6.5 \mathrm{~m}$ for wind and seawater). Kelvin attributed this to viscosity. However resonances like the one revealed by the second order theory are even stronger effects. In this section, we avoid such a resonance and assume that $K^{2} \neq \frac{1}{2}$. In view of describing the amplitude modulation of the progressive waves at the surface of the fluid, we need to proceed to the third-order solution, which yields the following nonlinear equation for the evolution of wave packets in $2+1$ dimensions,

$$
\begin{aligned}
& 2 \mathrm{i}\left(\frac{\partial A}{\partial t_{2}}+V_{l} \frac{\partial A}{\partial x_{2}}+V_{m} \frac{\partial A}{\partial y_{2}}\right)+P_{1} \frac{\partial^{2} A}{\partial x_{1}^{2}} \\
& +2 P_{2} \frac{\partial^{2} A}{\partial x_{1} \partial y_{1}}+P_{3} \frac{\partial^{2} A}{\partial y_{1}^{2}}=Q_{1}|A|^{2} A
\end{aligned}
$$


where

$$
\begin{aligned}
P_{1}= & \left(\alpha_{1} \sigma_{1}+\alpha_{2} \sigma_{2}\right)^{-1}\left\{2\left\lfloor\alpha_{1}\left(U^{(1)}\right)^{2}+\alpha_{2}\left(U^{(2)}\right)^{2}\right\rfloor\right. \\
& -K^{-1}\left(3 K^{2}+1\right)+l^{2} K^{-3}\left(1-3 K^{2}\right) \\
& \left.-4 V_{l}\left(\alpha_{1}\left(U^{(1)}\right)^{2}+\alpha_{2}\left(U^{(2)}\right)^{2}\right)-2 V_{l}^{2}-2 V_{A}^{2}\right\} \\
P_{2}= & \frac{\frac{l m}{K^{3}}\left(1-3 K^{2}\right)-2\left(\alpha_{1} U^{(1)}+\alpha_{2} U^{(2)}\right) V_{l}-2 V_{l} V_{m}}{\left(\alpha_{1} \sigma_{1}+\alpha_{2} \sigma_{2}\right)}, \\
P_{3}= & \frac{\frac{m^{2}}{K^{3}}\left(1-3 K^{2}\right)-\frac{1}{K}\left(1+3 K^{2}\right)}{\left(\alpha_{1} \sigma_{1}+\alpha_{2} \sigma_{2}\right)}, \\
Q_{1}= & \frac{1}{2}\left[4\left(2 K^{2}-1\right)^{-1}\left(\alpha_{1} \sigma_{1}^{2}-\alpha_{2} \sigma_{2}^{2}-K^{2} V_{A}^{2}\right)-4 K^{2}-K^{4}\right] .
\end{aligned}
$$

The PDE (18) is elliptic or hyperbolic, according to the sign of $P=\left(P_{2}^{2}-P_{1} P_{3}\right)$,

$$
\begin{aligned}
P=\left(\alpha_{1}\right. & \left.\sigma_{1}+\alpha_{2} \sigma_{2}\right)^{-2} \\
\times & {\left[\left(\frac{l m}{K^{3}}\left(1-3 K^{2}\right)-2\left(\alpha_{1} U^{(1)}+\alpha_{2} U^{(2)}\right) V_{l}-2 V_{l} V_{m}\right)^{2}\right.} \\
& -\left\{2\left[\alpha_{1}\left(U^{(1)}\right)^{2}+\alpha_{2}\left(U^{(2)}\right)^{2}\right]-K^{-1}\left(3 K^{2}+1\right)\right. \\
& +l^{2} K^{-3}\left(1-3 K^{2}\right)-4 V_{l}\left(\alpha_{1}\left(U^{(1)}\right)^{2}+\alpha_{2}\left(U^{(2)}\right)^{2}\right) \\
& \left.\left.-2 V_{l}^{2}-2 V_{A}^{2}\right\}\left\{\frac{m^{2}}{K^{3}}\left(1-3 K^{2}\right)-\frac{1}{K}\left(1+3 K^{2}\right)\right\}\right] .
\end{aligned}
$$

For the elliptic case, when $P$ is negative, we introduce the transformations

$\zeta_{1}=\frac{x_{1}}{P_{1}^{1 / 2}}, \quad \eta_{1}=\left(P_{3}-\frac{P_{2}^{2}}{P_{1}}\right)^{-1 / 2}\left(y_{1}-\frac{P_{2}}{P_{1}} x_{1}\right)$

Under such a transformation, (17) now reduces to a standard two-dimensional NLS equation. On rewriting (17) in the group-velocity reference frame, we obtain the elliptic equation

$2 \mathrm{i} \frac{\partial A}{\partial t_{2}}+\frac{\partial^{2} A}{\partial \zeta_{1}^{2}}+\frac{\partial^{2} A}{\partial \eta_{1}^{2}}=\frac{Q_{1}}{P_{1}}\left[\left(\alpha_{1} \sigma_{1}+\alpha_{2} \sigma_{2}\right) \rho_{1}\right]^{-1}|A|^{2} A_{0}$.

Putting

$Q \equiv Q\left(l, m, U, V_{A}^{2}\right)=\frac{Q_{1}}{P_{1}}\left[\left(\alpha_{1} \sigma_{1}+\alpha_{2} \sigma_{2}\right) \rho_{1}\right]^{-1}, \quad t_{2}=\tau$,

we obtain the elliptic equation as:

$2 \mathrm{i} \frac{\partial A}{\partial \tau}+\frac{\partial^{2} A}{\partial \zeta_{1}^{2}}+\frac{\partial^{2} A}{\partial \eta_{1}^{2}}=Q|A|^{2} A$

Equation (21) is the standard NLS equation in $2+1$ dimensions. For the hyperbolic case, when $P$ is positive, we introduce the transformations

$\zeta_{1}=\frac{x_{1}}{P_{1}^{1 / 2}}, \quad \eta_{1}=\left(\frac{P_{2}^{2}}{P_{1}}-P_{3}\right)^{-1 / 2}\left(\frac{P_{2}}{P_{1}} x_{1}-y_{1}\right)$.

Proceeding as before, we obtain the hyperbolic equation

$2 \mathrm{i} \frac{\partial A}{\partial \tau}+\frac{\partial^{2} A}{\partial \zeta_{1}^{2}}-\frac{\partial^{2} A}{\partial \eta_{1}^{2}}=Q|A|^{2} A$

Equations (21) and (23) can be expressed in the form

$2 \mathrm{i} \frac{\partial A}{\partial \tau}+\frac{\partial^{2} A}{\partial \zeta_{1}^{2}}+\Delta_{2} \frac{\partial^{2} A}{\partial \eta_{1}^{2}}=Q|A|^{2} A$

with $\quad \Delta_{2}=\left\{\begin{array}{rll}1 & \text { when } \quad P_{2}^{2}-P_{1} P_{2}<0, \\ -1 \quad \text { when } \quad & P_{2}^{2}-P_{1} P_{2}>0 .\end{array}\right.$

\section{General soliton solutions of two-dimensional NLS equation}

4.1. The elliptic case

Now we shall find classes of solutions of the standard twodimensional NLS equation, by applying the function transformation method. We search for solutions of the elliptic Eq. (21) in the form

$A=\theta\left(\tau, \zeta_{1}, \eta_{1}\right) \operatorname{expi}\left(c_{0} \tau+c_{1} \zeta_{1}+c_{2} \eta_{1}\right)$,

where $\theta\left(\tau, \zeta_{1}, \eta_{1}\right)=\theta^{*}\left(\tau, \zeta_{1}, \eta_{1}\right)$ and $c_{0}, c_{1}, c_{2}$ are real constants. By inserting (26) into (21), and putting $2 c_{0}+c_{1}^{2}+c_{2}^{2}=-C$ (constant), we get:

$2 \mathrm{i} \frac{\partial \theta}{\partial \tau}+2 \mathrm{i} c_{1} \frac{\partial \theta}{\partial \zeta_{1}}+2 \mathrm{i} c_{2} \frac{\partial \theta}{\partial \eta_{1}}+\frac{\partial^{2} \theta}{\partial \zeta_{1}^{2}}+\frac{\partial^{2} \theta}{\partial \eta_{1}^{2}}=Q \theta^{3}-C \theta$.

Using the function transformation

$\theta=\sqrt{\frac{C}{Q}} \sin \left(\frac{\varphi}{2}\right)$

where $\varphi$ is a function in $\tau, \zeta_{I}$ and $\eta_{I}$, we have

$2 \mathrm{i} \frac{\partial \theta}{\partial \tau}+2 \mathrm{i} c_{1} \frac{\partial \theta}{\partial \zeta_{1}}+2 \mathrm{i} c_{2} \frac{\partial \theta}{\partial \eta_{1}}$

$$
=\sqrt{\frac{C}{Q}} \cos (\varphi / 2)\left[i \frac{\partial \varphi}{\partial \tau}+i c_{1} \frac{\partial \varphi}{\partial \zeta_{1}}+i c_{2} \frac{\partial \varphi}{\partial \eta_{1}}\right],
$$

$\frac{\partial^{2} \theta}{\partial \zeta_{1}^{2}}=\frac{1}{2} \sqrt{\frac{C}{Q}}\left[\cos (\varphi / 2) \frac{\partial^{2} \varphi}{\partial \zeta_{1}^{2}}-\frac{1}{2} \sin (\varphi / 2)\left(\frac{\partial \varphi}{\partial \zeta_{1}}\right)^{2}\right]$,

$\frac{\partial^{2} \theta}{\partial \eta_{1}^{2}}=\frac{1}{2} \sqrt{\frac{C}{Q}}\left[\cos (\varphi / 2) \frac{\partial^{2} \varphi}{\partial \eta_{1}^{2}}-\frac{1}{2} \sin (\varphi / 2)\left(\frac{\partial \varphi}{\partial \eta_{1}}\right)^{2}\right]$,

$$
\begin{aligned}
Q \theta^{3}-C \theta & =Q\left(\frac{C}{Q}\right)^{3 / 2} \sin ^{3}(\varphi / 2)-C \sqrt{\frac{C}{Q}} \sin (\varphi / 2) \\
& =-\frac{C}{2} \sqrt{\frac{C}{Q}} \cos (\varphi / 2) \sin \varphi
\end{aligned}
$$


By substituting from (29) in Eq. (27) one gets

$$
\begin{aligned}
\mathrm{i} \frac{\partial \varphi}{\partial \tau} & +\mathrm{i} c_{1} \frac{\partial \varphi}{\partial \zeta_{1}}+\mathrm{i} c_{2} \frac{\partial \varphi}{\partial \eta_{1}}+\frac{1}{2}\left(\frac{\partial^{2} \varphi}{\partial \zeta_{1}^{2}}+\frac{\partial^{2} \varphi}{\partial \eta_{1}^{2}}\right) \\
& -\frac{1}{4} \tan (\varphi / 2)\left(\left(\frac{\partial \varphi}{\partial \zeta_{1}}\right)^{2}+\left(\frac{\partial \varphi}{\partial \eta_{1}}\right)^{2}\right)=-\frac{C}{2} \sin \varphi
\end{aligned}
$$

Setting $\varphi=\varphi(\zeta)$ which is a function of one variable $\zeta$ only (a function of the variables, see below), we easily see that

$\frac{\partial^{2} \varphi}{\partial \zeta_{1}^{2}}=\left(\frac{\partial \zeta}{\partial \zeta_{1}}\right)^{2} \frac{\mathrm{d}^{2} \varphi}{\mathrm{d} \zeta^{2}}+\frac{\partial^{2} \zeta}{\partial \zeta_{1}^{2}} \frac{\mathrm{d} \varphi}{\mathrm{d} \zeta}$

$\frac{\partial^{2} \varphi}{\partial \eta_{1}^{2}}=\left(\frac{\partial \zeta}{\partial \eta_{1}}\right)^{2} \frac{\mathrm{d}^{2} \varphi}{\mathrm{d} \zeta^{2}}+\frac{\partial^{2} \zeta}{\partial \eta_{1}^{2}} \frac{\mathrm{d} \varphi}{\mathrm{d} \zeta}$.

By substituting (31) into (30), we obtain

$$
\begin{aligned}
\left(\mathrm{i} \frac{\partial \zeta}{\partial \tau}\right. & \left.+\mathrm{i} c_{1} \frac{\partial \zeta}{\partial \zeta_{1}}+\mathrm{i} c_{2} \frac{\partial \zeta}{\partial \eta_{1}}+\frac{1}{2} \frac{\partial^{2} \zeta}{\partial \zeta_{1}^{2}}+\frac{1}{2} \frac{\partial^{2} \zeta}{\partial \eta_{1}^{2}}\right) \frac{\mathrm{d} \varphi}{\mathrm{d} \zeta} \\
& +\left[\left(\frac{\partial \zeta}{\partial \zeta_{1}}\right)^{2}+\left(\frac{\partial \zeta}{\partial \eta_{1}}\right)^{2}\right]\left[\frac{1}{2} \frac{\mathrm{d}^{2} \varphi}{\mathrm{d} \zeta^{2}}-\frac{1}{4} \tan (\varphi / 2)\left(\frac{\mathrm{d} \varphi}{\mathrm{d} \zeta}\right)^{2}\right] \\
& =-\frac{C}{2} \sin \varphi
\end{aligned}
$$

Explicitly, some solutions of (32) obey the following system of equations:

$\mathrm{i} \frac{\partial \zeta}{\partial \tau}+\mathrm{i} c_{1} \frac{\partial \zeta}{\partial \zeta_{1}}+\mathrm{i} c_{2} \frac{\partial \zeta}{\partial \eta_{1}}=\frac{\partial^{2} \zeta}{\partial \zeta_{1}^{2}}+\frac{\partial^{2} \zeta}{\partial \eta_{1}^{2}}=0$,

$\left(\frac{\partial \zeta}{\partial \zeta_{1}}\right)^{2}+\left(\frac{\partial \zeta}{\partial \eta_{1}}\right)^{2}=1$

$\frac{\mathrm{d}^{2} \varphi}{\mathrm{d} \zeta^{2}}=-\frac{C}{2} \sin \varphi, \quad \frac{\mathrm{d} \varphi}{\mathrm{d} \zeta}=\sqrt{2 C} \cos \left(\frac{\varphi}{2}\right)$.

Equation (34) is equivalent to a sine-Gordon equation, which has a well known soliton solution

$\varphi=4 \tan ^{-1} \exp \left(\sqrt{\frac{C}{2}} \zeta+\zeta_{0}\right)-\pi, \quad \zeta_{0}=$ cst.

From (35), (28) and (26), we obtain the corresponding soliton solution of the two-dimensional NLS equation (21) in the form

$$
\begin{aligned}
\theta & =\sqrt{\frac{C}{Q}} \tanh \left(\sqrt{\frac{C}{2}} \zeta+\zeta_{0}\right), \\
A & =\sqrt{\frac{C}{Q}} \tanh \left(\sqrt{\frac{C}{2}} \zeta+\zeta_{0}\right) \mathrm{e}^{\mathrm{i}\left(c_{0} \tau+c_{1} \zeta_{1}+c_{2} \eta_{1}\right)},
\end{aligned}
$$

where $\zeta$ denotes a solution of (33). As (33) has a lot of solutions, (36) contains a wide variety of interesting solitons of the two-dimensional NLS equation.
Now we intend to find a rather general solution of (33) in the form

$\zeta=F\left(\xi_{j}\right)+d_{0} \tau+d_{1} \zeta_{1}+d_{2} \eta_{1}$,

$\xi_{j}=b_{j 0} \tau+b_{j 1} \zeta_{1}+b_{j 2} \eta_{1}+\varepsilon_{j}$,

$d_{0}, d_{1}, d_{2}, b_{j 0}, b_{j 1}, b_{j 2}, \varepsilon_{j}=$ constants,

where $F\left(\xi_{j}\right)$ denotes an arbitrary function of $\xi_{j}$. Combining (33) and (37) we easily obtain

$$
\begin{aligned}
\mathrm{i} \frac{\partial \zeta}{\partial \tau} & +\mathrm{i} c_{1} \frac{\partial \zeta}{\partial \zeta_{1}}+\mathrm{i} c_{2} \frac{\partial \zeta}{\partial \zeta_{2}}=\mathrm{i}\left(b_{j 0}+c_{1} b_{j 1}+c_{2} b_{j 2}\right) \frac{\partial F}{\partial \xi_{j}} \\
& +\mathrm{i}\left(d_{0}+c_{1} d_{1}+c_{2} d_{2}\right),
\end{aligned}
$$

$\frac{\partial \zeta}{\partial \zeta_{1}}=b_{j 1} \frac{\partial F}{\partial \xi_{j}}+d_{1}, \quad \frac{\partial \zeta}{\partial \eta_{1}}=b_{j 2} \frac{\partial F}{\partial \xi_{j}}+d_{2}$,

$\left(\frac{\partial \zeta}{\partial \zeta_{1}}\right)^{2}+\left(\frac{\partial \zeta}{\partial \eta_{1}}\right)^{2}=\left[\left(b_{j 1}\right)^{2}+\left(b_{j 2}\right)^{2}\right]\left(\frac{\partial F}{\partial \xi_{j}}\right)^{2}$

$$
+\left(2 d_{1} b_{j 1}+2 d_{2} b_{j 2}\right) \frac{\partial F}{\partial \xi_{j}}+d_{1}^{2}+d_{2}^{2},
$$

$\frac{\partial^{2} \zeta}{\partial \zeta_{1}^{2}}=b_{j 1} b_{k 1} \frac{\partial^{2} F}{\partial \xi_{j} \partial \xi_{k}}, \quad \frac{\partial^{2} \zeta}{\partial \eta_{1}^{2}}=b_{j 2} b_{k 2} \frac{\partial^{2} F}{\partial \xi_{j} \partial \xi_{k}}$.

Requiring $F\left(\xi_{j}\right)$ to be arbitrary leads to the conditions

$b_{j 0}+c_{1} b_{j 1}+c_{2} b_{j 2}=0, \quad d_{0}+c_{1} d_{1}+c_{2} d_{2}=0$,

$b_{j 1} b_{k 1}+b_{j 2} b_{k 2}=0, \quad d_{1}^{2}+d_{2}^{2}=1$,

$d_{1} b_{j 1}+d_{2} b_{j 2}=0$.

The general solution (37) yields for (36) and (35) a rather general soliton solution of the two-dimensional NLS and sine-Gordon equations. They contain some interesting specific solutions, such as plane solitons, $N$ multiple solitons, propagational breathers and quadratic solitons. We will discuss them respectively below.

(a) Plane solitons

This is a simple case. By taking all $b_{j i}$ in (39) to be equal to zero, all $\xi_{j}$ become constant so that $F\left(\xi_{j}\right)=F\left(\varepsilon_{j}\right)=$ constants. Then (35) and (36) take the form

$$
\begin{aligned}
\varphi & =4 \tan ^{-1} \exp \left(\sqrt{\frac{C}{2}} \zeta+\zeta_{0}\right)-\pi, \quad \zeta_{0}=\text { cst. } \\
A & =\sqrt{\frac{C}{Q}} \tanh \left(\sqrt{\frac{C}{2}} \zeta+\zeta_{0}\right) \mathrm{e}^{\mathrm{i}\left(c_{0} \tau+c_{1} \zeta_{1}+c_{2} \eta_{1}\right)},
\end{aligned}
$$

where now $\zeta=d_{0} \tau+d_{1} \zeta_{1}+d_{2} \eta_{1}+$ constant. In this case, (40) and (41) denote hyperplanar soliton solutions of the two-dimensional NLS and sine-Gordon equations; this solution is stable.

(b) $N$ multiple soliton solutions

Let us select $F\left(\xi_{j}\right)$ in the form

$$
F\left(\xi_{j}\right)=\ln \sum_{j=1}^{N} \exp \left(b_{j 0} \tau+b_{j 1} \zeta_{1}+b_{j 2} \eta_{1}+\varepsilon_{j}\right) .
$$


Thus (37) yields:

$\zeta=\ln \left(\sum_{j=1}^{N} \exp \left[a_{j 0} \tau+a_{j 1} \zeta_{1}+a_{j 2} \eta_{1}+\varepsilon_{j}\right]\right)$

where $a_{j \alpha}=d_{\alpha}+b_{j \alpha}, \alpha=0,1,2$. The conditions (39) are simplified to

$a_{j 0}+c_{1} a_{j 1}+c_{2} a_{j 2}=0, \quad a_{j 1} a_{k 1}+a_{j 2} a_{k 2}=1$.

By direct calculation, we can easily prove that (43) is a solution of (33). Taking $j$ equal to $1,2, \ldots, N$ respectively yields

$\varphi=4 \tan ^{-1} \exp \left(\sqrt{\frac{C}{2}} \xi+\zeta_{0}\right)-\pi$

$A=\sqrt{\frac{C}{Q}} \tanh \left(\sqrt{\frac{C}{2}} \zeta+\zeta_{0}\right) \mathrm{e}^{\mathrm{i}\left(c_{0} \tau+c_{1} \zeta_{1}+c_{2} \eta_{1}\right)}$

where $\zeta$ is now given by (43). (45) and (46) are $N$ multiple soliton solutions of the two-dimensional NLS and sine-Gordon equations.

(c) Propagational breathers

Considering $N=2$ in (43) we get

$\zeta=\ln \left(\begin{array}{c}\exp \left(a_{10} \tau+a_{11} \zeta_{1}+a_{12} \eta_{1}+\varepsilon_{1}\right)+ \\ \exp \left(a_{20} \tau+a_{21} \zeta_{1}+a_{22} \eta_{1}+\varepsilon_{2}\right)\end{array}\right)$

which corresponds to a 2-soliton solution of the twodimensional NLS equation. For the $2+1$ dimensional case, we set

$a_{10}=-a_{20}, \quad a_{11}=-a_{21}, \quad a_{12}=a_{22}$,

$\varepsilon_{1}=\ln S, \quad \varepsilon_{2}=\ln S+\mathrm{i} \pi$.

Then (47) becomes

$\zeta=\ln \left[2 S \exp \left(a_{12} \eta_{1}\right) \sinh \left(a_{10} \tau+a_{11} \zeta_{1}\right)\right]$

and (44) gives the conditions

$a_{11}^{2}+a_{12}^{2}=1, \quad a_{10}+2 c_{1} a_{11}=0, \quad c_{2} a_{12}=0$.

If $a_{10}, a_{11}, S$ are some imaginary numbers such as

$a_{10}=\mathrm{i} a_{0}, \quad a_{11}=\mathrm{i} a_{1}, \quad 2 S=-\mathrm{i} B$

then (49) takes the form

$\zeta=\ln \left[B \exp \left(a_{12} \eta_{1}\right) \sin \left(a_{0} \tau+a_{1} \zeta_{1}\right)\right]$.

Summarizing again:

$\varphi=4 \tan ^{-1} \exp \left(\sqrt{\frac{C}{2}} \zeta+\zeta_{0}\right)-\pi$

$A=\sqrt{\frac{C}{Q}} \tanh \left(\sqrt{\frac{C}{2}} \zeta+\zeta_{0}\right) \mathrm{e}^{\mathrm{i}\left(c_{0} \tau+c_{1} \zeta_{1}+c_{2} \eta_{1}\right)}$,

where now $\zeta$ is given by (52). Thus (53) and (54) are a two dimensional breather which propagates in the $\zeta_{1}$ direction and has a constant value on the straight line $a_{12} \eta_{1}=$ constant. This is an interesting result.

(d) Quadratic solitons

We take the solution (33) in the form

$\zeta=\left(b_{j 0} \tau+b_{j 1} \zeta_{1}+b_{j 2} \eta_{1}+\varepsilon_{j}\right)^{2}+d_{0} \tau+d_{1} \zeta_{1}+d_{2} \eta_{1}$,

or

$$
\begin{aligned}
\zeta & =b_{j 0} b_{j 0} \tau^{2}+b_{j 1} b_{j 1} \zeta_{1}^{2}+b_{j 2} b_{j 2} \eta_{1}^{2}+2 b_{j 1} b_{j 2} \zeta_{1} \eta_{1} \\
& +\left(2 \varepsilon_{j} b_{j 1}+2 b_{j 0} b_{j 1} \tau+d_{1}\right) \zeta_{1}+\left(2 \varepsilon_{j} b_{j 2}+2 b_{j 0} b_{j 2} \tau+d_{2}\right) \eta_{1} \\
& +\left(2 \varepsilon_{j} b_{j 0}+d_{0}\right) \tau+\varepsilon_{j} \varepsilon_{j}, \quad j=1,2, \ldots, N
\end{aligned}
$$

It describes some general quadratic solutions at any definite time. These quadratic solitons include all specific ones such as:

(i) Circular soliton

By choosing the constants of (55) so that

$b_{j i} b_{j k}=\delta_{i k}, \quad j=1,2, \ldots, N$,

(55) becomes

$$
\begin{aligned}
\zeta= & \tau^{2}+\zeta_{1}^{2}+\eta_{1}^{2}+\left(2 \varepsilon_{j} b_{j 1}+d_{1}\right) \zeta_{1}+\left(2 \varepsilon_{j} b_{j 2}+d_{2}\right) \eta_{1} \\
& +\left(2 \varepsilon_{j} b_{j 0}+d_{0}\right) \tau+\varepsilon_{j} \varepsilon_{j} .
\end{aligned}
$$

Thus we obtain a circular soliton with radius

$$
\begin{aligned}
R= & {\left[\left(\varepsilon_{j} b_{j i}+\frac{d_{i}}{2}\right)\left(\varepsilon_{k} b_{k i}+\frac{d_{i}}{2}\right)\right.} \\
& \left.-\tau^{2}-\left(2 \varepsilon_{j} b_{j 0}+d_{0}\right) \tau-\varepsilon_{j} \varepsilon_{j}\right]^{1 / 2},
\end{aligned}
$$

and with center

$$
G\left(-2 \varepsilon_{j} b_{j 1}-2 b_{j 0} b_{j 1} \tau-d_{1},-2 \varepsilon_{j} b_{j 2}-2 b_{j 0} b_{j 2} \tau-d_{2}\right) .
$$

This moves along a space line. Thus (57), (35) and (36) give some circularly shaped solitons of the two-dimensional NLS and sine-Gordon equations.

(ii) Elliptical solitons

Under the conditions

$b_{j 1} b_{j 1}>0, \quad b_{j 2} b_{j 2}>0$,

$b_{j i} b_{j k}=0, \quad i \neq k, \quad j=1,2, \ldots, N$,

then (55) becomes

$$
\begin{aligned}
\zeta= & b_{j 0} b_{j 0} \tau^{2}+b_{j 1} b_{j 1} \zeta_{1}^{2}+b_{j 2} b_{j 2} \eta_{1}^{2}+\left(2 \varepsilon_{j} b_{j 1}+d_{1}\right) \zeta_{1} \\
& +\left(2 \varepsilon_{j} b_{j 2}+d_{2}\right) \eta_{1}+\left(2 \varepsilon_{j} b_{j 0}+d_{0}\right) \tau+\varepsilon_{j} \varepsilon_{j} \\
& j=1,2, \ldots, N
\end{aligned}
$$

Equation (61) are ellipses and (35) and (36) are elliptical solitons. 
(iii) Hyperbolic solitons

Let the constants satisfy

$b_{j 1} b_{j 1}>0, \quad b_{j 2} b_{j 2}<0, \quad b_{j i} b_{j k}=0, \quad i \neq k$

$j=1,2, \ldots, N$.

From (55), we obtain hyperbolas and (35) and (36) give the corresponding hyperbolic shape solitons of two-dimensional NLS and sine-Gordon equations.

\subsection{The hyperbolic case}

We search now solutions of the hyperbolic equation (23) in the form

$A=\Phi\left(\tau, \zeta_{1}, \eta_{1}\right) \operatorname{expi}\left(n_{0} \tau+n_{1} \zeta_{1}+n_{2} \eta_{1}\right)$

where $\Phi\left(\tau, \zeta_{1}, \eta_{1}\right)=\Phi^{*}\left(\tau, \zeta_{1}, \eta_{1}\right)$ and $n_{0}, n_{1}, n_{2}$ are real constants.

By inserting (63) into (23), one obtains putting $2 n_{0}+n_{1}^{2}-n_{2}^{2}=-C^{\prime}$ (constant)

$2 \mathrm{i} \frac{\partial \Phi}{\partial \tau}+2 \mathrm{i} n_{1} \frac{\partial \Phi}{\partial \zeta_{1}}-2 \mathrm{i} n_{2} \frac{\partial \Phi}{\partial \eta_{1}}+\frac{\partial^{2} \Phi}{\partial \zeta_{1}^{2}}-\frac{\partial^{2} \Phi}{\partial \eta_{1}^{2}}=Q \Phi^{3}-C^{\prime} \Phi$

Let us make a function transformation

$\Phi=\sqrt{\frac{C^{\prime}}{Q}} \sin \left(\frac{\Psi}{2}\right)$

where $\Psi$ is a function in $\tau, \zeta_{1}$ and $\eta_{1}$. Calculation of the terms in (64) yields

$2 \mathrm{i} \frac{\partial \Phi}{\partial \tau}+2 \mathrm{i} n_{1} \frac{\partial \Phi}{\partial \zeta_{1}}-2 \mathrm{i} n_{2} \frac{\partial \Phi}{\partial \eta_{1}}$

$=\sqrt{\frac{C^{\prime}}{Q}} \cos (\Psi / 2)\left[\mathrm{i} \frac{\partial \Psi}{\partial \tau}+\mathrm{i} n_{1} \frac{\partial \Psi}{\partial \zeta_{1}}-\mathrm{i} n_{2} \frac{\partial \Psi}{\partial \eta_{1}}\right]$,

$\frac{\partial^{2} \Phi}{\partial \zeta_{1}^{2}}=\frac{1}{2} \sqrt{\frac{C^{\prime}}{Q}}\left[\cos (\Psi / 2) \frac{\partial^{2} \Psi}{\partial \zeta_{1}^{2}}-\frac{1}{2} \sin (\Psi / 2)\left(\frac{\partial \Psi}{\partial \zeta_{1}}\right)^{2}\right]$,

$\frac{\partial^{2} \Phi}{\partial \eta_{1}^{2}}=\frac{1}{2} \sqrt{\frac{C^{\prime}}{Q}}\left[\cos (\Psi / 2) \frac{\partial^{2} \Psi}{\partial \eta_{1}^{2}}-\frac{1}{2} \sin (\Psi / 2)\left(\frac{\partial \Psi}{\partial \eta_{1}}\right)^{2}\right]$,

$Q \Phi^{3}-C^{\prime} \Phi=-\frac{C^{\prime}}{2} \sqrt{\frac{C^{\prime}}{Q}} \cos (\Psi / 2) \sin \Psi$

By substituting from (66) in Eq. (64), we get

$$
\begin{aligned}
\mathrm{i} \frac{\partial \Psi}{\partial \tau} & +\mathrm{i} n_{1} \frac{\partial \Psi}{\partial \zeta_{1}}-\mathrm{i} n_{2} \frac{\partial \Psi}{\partial \eta_{1}}+\frac{1}{2}\left(\frac{\partial^{2} \Psi}{\partial \zeta_{1}^{2}}-\frac{\partial^{2} \Psi}{\partial \eta_{1}^{2}}\right) \\
& -\frac{1}{4} \tan (\Psi / 2)\left(\left(\frac{\partial \Psi}{\partial \zeta_{1}}\right)^{2}-\left(\frac{\partial \Psi}{\partial \eta_{1}}\right)^{2}\right)=-\frac{C^{\prime}}{2} \sin \Psi
\end{aligned}
$$

Setting $\Psi=\Psi(\zeta)$ which is a function of one function $\zeta$ only and by substituting from (31) into (67), we easily see that

$$
\begin{aligned}
\left(\mathrm{i} \frac{\partial \zeta}{\partial \tau}\right. & \left.+\mathrm{i} n_{1} \frac{\partial \zeta}{\partial \zeta_{1}}-\mathrm{i} n_{2} \frac{\partial \zeta}{\partial \eta_{1}}+\frac{1}{2} \frac{\partial^{2} \zeta}{\partial \zeta_{1}^{2}}-\frac{1}{2} \frac{\partial^{2} \zeta}{\partial \eta_{1}^{2}}\right) \frac{\mathrm{d} \Psi}{\mathrm{d} \zeta} \\
& +\left[\left(\frac{\partial \zeta}{\partial \zeta_{1}}\right)^{2}-\left(\frac{\partial \zeta}{\partial \eta_{1}}\right)^{2}\right]\left[\frac{1}{2} \frac{\mathrm{d}^{2} \Psi}{\mathrm{d} \zeta^{2}}-\frac{1}{4} \tan (\Psi / 2)\left(\frac{\mathrm{d} \Psi}{\mathrm{d} \zeta}\right)^{2}\right] \\
& =-\frac{C^{\prime}}{2} \sin \Psi
\end{aligned}
$$

Some solutions of (68) obey the following system of equations:

$\mathrm{i} \frac{\partial \zeta}{\partial \tau}+\mathrm{i} n_{1} \frac{\partial \zeta}{\partial \zeta_{1}}-\mathrm{i} n_{2} \frac{\partial \zeta}{\partial \eta_{1}}=\frac{\partial^{2} \zeta}{\partial \zeta_{1}^{2}}-\frac{\partial^{2} \zeta}{\partial \eta_{1}^{2}}=0$,

$\left(\frac{\partial \zeta}{\partial \zeta_{1}}\right)^{2}-\left(\frac{\partial \zeta}{\partial \eta_{1}}\right)^{2}=1$

$\frac{\mathrm{d}^{2} \Psi}{\mathrm{d} \zeta^{2}}=-\frac{C^{\prime}}{2} \sin \Psi, \quad \frac{\mathrm{d} \Psi}{\mathrm{d} \zeta}=\sqrt{2 C^{\prime}} \cos \left(\frac{\Psi}{2}\right)$

Equation (70) is equivalent to a sine-Gordon equation, which has the well known soliton solution

$\Psi=4 \tan ^{-1} \exp \left(\sqrt{\frac{C^{\prime}}{2}} \zeta+\zeta_{0}\right)-\pi, \quad \zeta_{0}=$ constant

Applying (71) and (65) to (63), we obtain a soliton solution of the two-dimensional NLS equation (24) in the form

$$
\begin{aligned}
& \Phi=\sqrt{\frac{C^{\prime}}{Q}} \tanh \left(\sqrt{\frac{C^{\prime}}{2}} \zeta+\zeta_{0}\right), \\
& A=\sqrt{\frac{C}{Q}} \tanh \left(\sqrt{\frac{C}{2}} \zeta+\zeta_{0}\right) \mathrm{e}^{\mathrm{i}\left(c_{0} \tau+c_{1} \zeta_{1}+c_{2} \eta_{1}\right)},
\end{aligned}
$$

where $\zeta$ denotes a solution of (70). As (70) has a large amount of solutions, (72) contains a vast variety of interesting solitons of the two-dimensional NLS equation.

Now we search for a kind of general solution of (67) in the form

$\zeta=F\left(\xi_{j}\right)+m_{0} \tau+m_{1} \zeta_{1}+m_{2} \eta_{1}$,

$\xi_{j}=b_{j 0} \tau+b_{j 1} \zeta_{1}+b_{j 2} \eta_{1}+\varepsilon_{j}$,

$m_{0}, m_{1}, m_{2}, b_{j 0}, b_{j 1}, b_{j 2}, \varepsilon_{j}=$ constants

where $F\left(\xi_{j}\right)$ denotes an arbitrary function of $\xi_{j}$. Combining (70) and (73) we easily obtain

$$
\begin{aligned}
& \mathrm{i} \frac{\partial \zeta}{\partial \tau}+\mathrm{i} n_{1} \frac{\partial \zeta}{\partial \zeta_{1}}-\mathrm{i} n_{2} \frac{\partial \zeta}{\partial \zeta_{2}}=\mathrm{i}\left(m_{0}+n_{1} m_{1}-n_{2} m_{2}\right) \\
& \quad+\mathrm{i}\left(b_{j 0}+n_{1} b_{j 1}-n_{2} b_{j 2}\right) \frac{\partial F}{\partial \xi_{j}}
\end{aligned}
$$




$$
\begin{aligned}
\left(\frac{\partial \zeta}{\partial \zeta_{1}}\right)^{2}+\left(\frac{\partial \zeta}{\partial \eta_{1}}\right)^{2} & \\
= & {\left[\left(b_{j 1}\right)^{2}-\left(b_{j 2}\right)^{2}\right]\left(\frac{\partial F}{\partial \xi_{j}}\right)^{2}+\left(2 m_{1} b_{j 1}-2 m_{2} b_{j 2}\right) \frac{\partial F}{\partial \xi_{j}} } \\
& +m_{1}^{2}-m_{2}^{2}=1, \\
\frac{\partial^{2} \zeta}{\partial \zeta_{1}^{2}}= & b_{j 1} b_{k 1} \frac{\partial^{2} F}{\partial \xi_{j} \partial \xi_{k}}=0, \quad \frac{\partial^{2} \zeta}{\partial \eta_{1}^{2}}=b_{j 2} b_{k 2} \frac{\partial^{2} F}{\partial \xi_{j} \partial \xi_{k}}=0
\end{aligned}
$$

Requiring $F\left(\xi_{j}\right)$ to be arbitrary leads to the conditions

$b_{j 0}+n_{1} b_{j 1}-n_{2} b_{j 2}=0, \quad m_{0}+n_{1} m_{1}-n_{2} m_{2}=0$

$b_{j 1} b_{k 1}-b_{j 2} b_{k 2}=0, \quad m_{1} m_{1}-m_{2} m_{2}=1$,

$m_{1} b_{j 1}-m_{2} b_{j 2}=0$.

The general solution (73) yields for (72) and (71) a rather general soliton solution of the two-dimensional NLS and sine-Gordon equations. They contain some interesting specific solutions, such as plane solitons, $N$ multiple solitons, propagational breathers and quadratic solitons. We will discuss them respectively below.

(a) The plane solitons

This is a simple case. By taking all $b_{j i}$ in (75) to be equal to zero, $F\left(\xi_{j}\right)$ becomes a constant so that $b_{j 0}=b_{j 1}=b_{j 2}=0, F\left(\xi_{j}\right)=F\left(\varepsilon_{j}\right)=$ constants.

Summarizing we have (71) and (72)

$$
\Psi=4 \tan ^{-1} \exp \left(\sqrt{\frac{C^{\prime}}{2}} \zeta+\zeta_{0}\right)-\pi, \quad \zeta_{0}=\text { const }
$$

$$
A=\sqrt{\frac{C}{Q}} \tanh \left(\sqrt{\frac{C}{2}} \zeta+\zeta_{0}\right) \mathrm{e}^{\mathrm{i}\left(c_{0} \tau+c_{1} \zeta_{1}+c_{2} \eta_{1}\right)},
$$

where $\zeta=m_{0} \tau+m_{1} \zeta_{1}+m_{2} \eta_{1}+$ constant. In this case, (76) and (77) represent a hyperplanar soliton solution of the two-dimensional NLS and sine-Gordon equations. This solution is stable.

(b) $N$ multiple soliton solutions

Let us select $F\left(\xi_{j}\right)$ in the form

$F\left(\xi_{j}\right)=\ln \sum_{j=1}^{N} \exp \left(b_{j 0} \tau+b_{j 1} \zeta_{1}+b_{j 2} \eta_{1}+\varepsilon_{j}\right)$.

Application of (73) and (78) leads to

$\zeta=\ln \left(\sum_{j=1}^{N} \exp \left[D_{j 0} \tau+D_{j 1} \zeta_{1}+D_{j 2} \eta_{1}+\varepsilon_{j}\right]\right)$

Here $D_{j \alpha}=m_{\alpha}+b_{j \alpha}, \alpha=0,1,2$. The conditions (75) are simplified to

$$
D_{j 0}+n_{1} D_{j 1}-n_{2} D_{j 2}=0, \quad D_{j 1} D_{k 1}-D_{j 2} D_{k 2}=1 .
$$

By direct calculation, we can easily prove that (79) is a solution of (69). Taking $j$ equal to $1,2, \ldots, N$, respectively, (79), (71) and (72) gives $N$ multiple soliton solutions of the two-dimensional NLS and sineGordon equations.

(c) Propagational breathers

Considering $N=2$ in (79) we get

$\zeta=\ln \left(\begin{array}{c}\exp \left(D_{10} \tau+D_{11} \zeta_{1}+D_{12} \eta_{1}+\varepsilon_{1}\right)+ \\ \exp \left(D_{20} \tau+D_{21} \zeta_{1}+D_{22} \eta_{1}+\varepsilon_{2}\right)\end{array}\right)$

which corresponds to a 2-soliton of the two-dimensional NLS equation. For the $2+1$ dimensional case, we set

$$
\begin{aligned}
& D_{10}=-D_{20}, \quad D_{11}=-D_{21}, \quad D_{12}=D_{22}, \\
& \varepsilon_{1}=\ln S, \quad \varepsilon_{2}=\ln S+\mathrm{i} \pi .
\end{aligned}
$$

Then (73) becomes

$\zeta=\ln \left[2 S \exp \left(D_{12} \eta_{1}\right) \sinh \left(D_{10} \tau+D_{11} \zeta_{1}\right)\right]$

and (80) gives the conditions

$D_{11}^{2}-D_{12}^{2}=1, \quad D_{10}+2 n_{1} D_{11}=0, \quad n_{2} D_{12}=0$

Taking $D_{10}, D_{11}, S$ as imaginary numbers

$D_{10}=\mathrm{i} D_{0}, \quad D_{11}=\mathrm{i} D_{1}, \quad 2 S=\mathrm{i} \lambda$,

then (49) takes the form

$\zeta=D_{12} \eta_{1}+\ln \left[\lambda \sin \left(D_{0} \tau+D_{1} \zeta_{1}\right)\right]$

Then (71), (72) and (86) are a two-dimensional breather which propagates in the $\zeta_{1}$ direction and has a constant value on the straight line $D_{12} \eta_{1}=$ constant. This is an interesting result.

(d) Quadratic solitons

We take the solution (69) in the form:

$\zeta=\left(b_{j 0} \tau+b_{j 1} \zeta_{1}+b_{j 2} \eta_{1}+\varepsilon_{\mathrm{j}}\right)^{2}+m_{0} \tau+m_{1} \zeta_{1}+m_{2} \eta_{1}$,

$j=1,2, \ldots, N$.

It describes some general quadratic surfaces at any definite time. These quadratic solutions include all specific ones such as:

(i) The circular soliton

By choosing the constants of (87) as in (56), (87) becomes

$$
\begin{aligned}
\zeta= & \tau^{2}+\zeta_{1}^{2}+\eta_{1}^{2}+\left(2 \varepsilon_{j} b_{j 1}+m_{1}\right) \zeta_{1} \\
& +\left(2 \varepsilon_{j} b_{j 2}+m_{2}\right) \eta_{1}+\left(2 \varepsilon_{j} b_{j 0}+m_{0}\right) \tau+\varepsilon_{j} \varepsilon_{j},
\end{aligned}
$$

which moves along a space line. Thus (88), (71) and (72) give circular solitons which are solutions of the two-dimensional NLS and sine-Gordon equations. 
(ii) Elliptical solitons

Under the conditions (60), (87) becomes

$$
\begin{aligned}
\zeta= & b_{j 0} b_{j 0} \tau^{2}+b_{j 1} b_{j 1} \zeta_{1}^{2}+b_{j 2} b_{j 2} \eta_{1}^{2}+\left(2 \varepsilon_{j} b_{j 1}+m_{1}\right) \zeta_{1} \\
& +\left(2 \varepsilon_{j} b_{j 2}+m_{2}\right) \eta_{1}+\left(2 \varepsilon_{j} b_{j 0}+m_{0}\right) \tau+\varepsilon_{j} \varepsilon_{j} \\
& j=1,2, \ldots, N
\end{aligned}
$$

Equation (89) represents ellipses and (71) and (72) are thus elliptical shaped solitons.

(iii) Hyperbolic solitons

Let the constants satisfy (62), then from (87), we obtain some hyperbolas and (71) and (72) give the corresponding hyperbolic shaped solitons of the two-dimensional NLS and sine-Gordon equations.

\section{Soliton stability}

Equation (24) admits two-dimensional soliton solutions traveling at any acute angle relative to the group velocity of the wave packet. The amplitude of the traveling wave depends on both $\eta$ and $\zeta$. There may be a crossover from one region to another. Now we shall discuss the stability of the solutions of (24). Following Zakharov [32] and Berge [43], Eq. (24) can be obtained by deriving the functional relation $\delta L / \delta A^{*}=0$ from the Lagrangian

$L=\frac{1}{2} \mathrm{i} \iint\left(A^{*} \frac{\partial A}{\partial \tau}-A \frac{\partial A^{*}}{\partial \tau}\right) \mathrm{d} \zeta_{1} \mathrm{~d} \eta_{1}-H$,

where $A^{*}$ denotes the complex conjugate function of $A$ where $H$ and $N$ are two integrals of motion for the elliptic case $P<0$ as:

$N=\iint|A|^{2} \mathrm{~d} \zeta_{1} \mathrm{~d} \eta_{1}$

$H=\frac{1}{2} \iint\left(\Delta_{1}\left|\frac{\partial A}{\partial \zeta_{1}}\right|^{2}+\Delta_{2}\left|\frac{\partial A}{\partial \eta_{1}}\right|^{2}+\frac{Q}{2}|A|^{2}\right) \mathrm{d} \zeta_{1} \mathrm{~d} \eta_{1}$,

where the normalization $N$ is the wave action and $H$ is the Hamiltonian. Furthermore, one can show by direct calculation that (91) and (92) in (24) yields the virial equation

$\frac{\partial^{2} I}{\partial \tau^{2}}=\frac{\partial^{2}}{\partial \tau^{2}} \iint\left(\zeta_{1}^{2}+\eta_{1}^{2}\right)|A|^{2} \mathrm{~d} \zeta_{1} \mathrm{~d} \eta_{1}=4 H$

Here $I$, the moment of inertia of the wave packet, is

$I=\iint\left(\zeta_{1}^{2}+\eta_{1}^{2}\right)|A|^{2} \mathrm{~d} \zeta_{1} \mathrm{~d} \eta_{1}$

since $H$ is a constant of motion. Therefore (93) may be integrated twice, and making use of (94), we have

$\langle I\rangle=\frac{2 \tau^{2}}{N} H+\gamma_{1} \tau+\gamma_{2}$, where the average value of the moment of inertia $I$ is given by

$\langle I\rangle=\frac{I}{N}$

and $\gamma_{1}$ and $\gamma_{2}$ are constants of integration given by the initial data:

$\gamma_{1}=\left.\frac{\partial\langle I\rangle}{\partial \zeta_{1}}\right|_{\tau=0} \quad$ and $\quad \gamma_{2}=\left.\langle I\rangle\right|_{\tau=0}$

It should be noted that $\gamma_{2}$ is always positive. A sufficient condition for the finite-time collapse of $I$ to zero is $H<0$. Here $|A| \rightarrow 0$ everywhere except at the collapse point. The value of $|A|$ tends to infinity at the collapse point at the same time, due to the invariance of $N$. This implies that the $L^{2}$ norm of the solution is preserved as the collapse of its support proceeds. $H$ can only be negative when $Q<0$. It is possible to find certain initial conditions rendering $Q<0$, implying $H<0$. In particular, if $\gamma_{1}(0)=0$ then $\langle I\rangle \rightarrow 0$ as $\tau$ approaches the time of collapse, $\tau_{0}=\left(\gamma_{2} N / 2 H\right)^{1 / 2}$, to a point in the $\left(\zeta_{1}, \eta_{1}\right)$ plane. If, however, $H>0$ then an additional forcing of the initial data is required for collapse to occur. However $\langle I\rangle \rightarrow 0$ in a finite time requires $\gamma_{1}<0$ and $\gamma_{1}^{2} \geq(8 H / N) \gamma_{2}$. Following Berkshire and Gibbon [44], two important results on collapse are

(a) if a collapse occurs then it must do so in a finite time;

(b) a collapse cannot occur unless the total angular momentum is zero.

We now define the angular momentum $J$ as

$$
\begin{aligned}
J= & \iint\left(\zeta_{1}, \eta_{1}\right) \\
& \times\left(\frac{\Delta_{1}}{2 \mathrm{i}}\left(A^{*} \frac{\partial A}{\partial \zeta_{1}}-A \frac{\partial A^{*}}{\partial \zeta_{1}}\right), \frac{\Delta_{2}}{2 \mathrm{i}}\left(A^{*} \frac{\partial A}{\partial \eta_{1}}-A \frac{\partial A^{*}}{\partial \eta_{1}}\right)\right) \mathrm{d} \zeta_{1} \mathrm{~d} \eta_{1} .
\end{aligned}
$$

Total wave collapse will take place provided the blow-up singularity does not occur at times earlier than the collapse predicted by the virial theorem. Rasmussen and Rypdal [45] and Berge [43], have shown that the virial theorem cannot be used to predict the time of blow up, and it fails to give information about whether or not $\langle I\rangle$ is concentrated at the singular point. In an actual physical case, the dissipative effects will inhibit the earlier collapse from occurring. However, Berge [43] has shown that collapse will not take place in the hyperbolic two-dimensional case. It is interesting to note here that the corresponding Eq. (92) for the Hamiltonian $H$ does not contain any nonlinear term for the hyperbolic case. We should like to point out here that for collapse to occur in finite time, the group-velocity rate and the nonlinear interaction coefficient $Q$ must change sign as the quantities $\rho, l, m$ and $V_{A}$ are varied.

\section{Discussion and summary}

It has been shown that the evolution of the amplitude of the progressive waves in superposed fluids, in the presence of a magnetic field, is governed by a $2+1$ dimensional 
NLS equation. It was demonstrated that the waves can be unstable against modulation [34]. In this paper, we examined the Kelvin-Helmholtz MHD flow in the subcritical regime, when the velocity difference $U$ is less than the critical velocity $U_{c}$. The phenomenon of nonlinear internal resonance for the case where fundamental and second-harmonic modes travel with the same phase velocity is investigated. A special scaling is used to determine the nonlinear interaction coefficients. The basic equations governing the MHD flow are given; and the standard NLS equations in $2+1$ dimensions in elliptic and hyperbolic cases are rederived. We found soliton solutions to the standard NLS equation in $2+1$ dimensions. We applied the function transformation method, thus the twodimensional NLS equation is transformed to a sineGordon equation, which depends only on one function, $\zeta$. We obtained rather general classes of solutions of the equation in $\zeta$ which leads to rather general soliton solutions of NLS. The latter contains quite interesting specific solutions, such as $N$ multiple solitons, propagational breathers and quadratic solitons. Wu et al. [19-20] studied the solitary waves and discussed the possibility for applying their results to nonlinear waves observed by the Freja scientific satellite in the upper ionosphere [21]. Finally, the stability of the solution of the standard NLS equation in $2+1$ dimensions is discussed. The present analysis was applied by us previously to nonlinear dispersive instabilities in Rayleigh-Taylor magnetohydrodynamic flows [46].

\section{References}

1. Blandford, R. D., Begelman, M. C. and Rees, M. J., Sci. Am. 246, 84 (1982).

2. Parhi, S., Phys. Fluids. B4, 1589 (1992).

3. Schilinski, A. Yu. and Chernii, G. G., "Nonlinear Wave Processes," (Moscow, Mir. 1987).

4. Dungey, J. W. in "Proceedings of the Ionosphere," (The Physical Society of London, 1955) p. 225.

5. Min, K. W., Kim, T. and Lee, H., Planet. Space Sci. 45, 495 (1997).

6. Vranješ, J., Planet. Space Sci. 47, 1531 (1999).

7. Parker, E. N., "Interplanetary Dynamical Processes." (New York, 1963).

8. Pécseli, H. L., Rasmussen, J. J., Sugai, H. and Thomsen, K., Plasma Phys. Contr. Fusion 26, 1021 (1984).

9. Pécseli, H. L., Rasmussen, J. J. and Thomsen, K., Phys. Rev. Lett. 52, 2148 (1984).

10. Pécseli, H. L., Rasmussen, J. J. and Thomsen, K., Plasma Phys. Contr. Fusion 27, 837 (1985).
11. Huld, T., Iizuka, S., Pécseli, H. L. and Rasmussen, J. J., Plasma Phys. Contr. Fusion 30, 1297 (1988).

12. Huld, T., Nielsen, A. H., Pécseli, H. L. and Rasmussen, J. J., Phys. Fluids B3, 1609 (1991).

13. Nielsen, A. H., Pécseli, H. L. and Rasmussen, J. J., Ann. Geophys. 10, 655 (1992).

14. Mikhailovskii, A. B., "Theory of Plasma Instabilities," Vol. 2 (Moscow, Atomizdat, 1971).

15. Wang, Z., Pritchett P. L. and Ashour-Abdalla, M., Phys. Fluids. B4, 1092 (1992).

16. Zhdanov, S. K. and Prudkoi, Yu. V., Fizika plasmi 19, 433 (1993).

17. Lindqvist, P. A., Marklund, G. T. and Blomberg, L. G., Space Sci. Rev. 70, 593 (1994).

18. Temerin, M., Woldorff, M. and Mozer, S. F., Phys. Rev. Lett. 43, 1941 (1979).

19. Wu, D. J., Yu, D. Y. and Falthammar, C. G., Phys. Plasmas, 2, 4467 (1995).

20. Wu, D. J., Huang, G. L., Yu, D. Y. and Falthammar, C. G., Phys. Plasmas 3, 2879 (1996).

21. Holback, B. et al., Space Sci. Rev. 70, 577 (1994).

22. Michael, D. H., Proc. Camb. Phil. Soc. 51, 528 (1955).

23. Chandrasekhar, S., "Hydrodynamic and Hydromagnetic Stability," (Clarendon Press, Oxford, 1961).

24. Drazin, P. G., J. Fluid Mech. 42, 321 (1970).

25. Nayfeh, A. H. and Saric, W. S., J. Fluid Mech. 46, 209 (1971).

26. Weissman, M. A., Phil. Trans. R. Soc. Lond. A290, 639 (1979).

27. Kant, R. S. and Malik, S. K., Astrophys. Space Sci. 86, 345 (1982).

28. Malik, S. K. and Singh, M., Astrophys. Space Sci. 109, 231 (1985).

29. Zakharov, V. E. and Shabat, A. B., Soviet Phys. JETP 43, 62 (1972).

30. Khater, A. H., Callebaut, D. K. and El-Kalaawy, O. H., I1 Nuovo Cimento 103B, 1121 (1998).

31. Zakharov, V. E. and Synakh, S., Soviet Phys. JETP 41, 465 (1975).

32. Zakharov, V. E., Soviet Phys. JETP 14, 908 (1972).

33. Gibbon, J. D. and McGuinness, M. J., Phys. Lett. 77A, 118 (1980).

34. Singh, M., Khosla, H. K. and Malik, S. K., J. Plasma Phys. 59, 27 (1998).

35. Hirota, R., in "Bäcklund Transformations" (Edited by R. Bullough and P. Caudret) (Springer-Verlag, Berlin), p. 157 (1980).

36. Coffey, M., SIAM J. Appl. Math. 50, 1580 (1990).

37. Kudryashov, N., Phys. Lett. A155, 269 (1991).

38. Hereman, W. and Takaoka, M., J. Phys. A: Math. Gen. 23, 4805 (1990).

39. Jeffrey, A. and Mohammad, M., Wave Motion 14, 369 (1990).

40. Jeffrey, A. and Xu, S., Wave Motion 11, 559 (1989).

41. Nayfeh, A. H., "Perturbation Methods," (Wiley-Interscience Publication, New York, 1973).

42. Pusri, A. and Malik, S. K., J. Plasma Phys. 53, 145 (1995).

43. Berge, L., Phys. Lett. 189A, 290 (1994).

44. Berkshire, F. H. and Gibbon, J. D., Stud. Appl. Maths. 69, 229 (1983).

45. Rasmussen, J. J. and Rypdal, K., Physica Scripta 33, 481 (1986).

46. Khater, A. H., Callebaut, D. K., Malfliet, W. and Seadawy, A. R., Physica Scripta 64, 533 (2001). 